\title{
Bluetooth Controlled Arduino Based Robotic Arm
}

\author{
Srija Dasgupta \\ Computer Science and Engineering of TINT \\ (MAKAUT) \\ Techno International Newtown (MAKAUT) \\ Kolkata, India \\ mamandasgupta@gmail.com
}

\author{
Avirup Ghosh \\ Computer Science and Engineering of TINT \\ (MAKAUT) \\ Techno International Newtown (MAKAUT) \\ Kolkata, India \\ ghoshavirup0@gmail.com
}

\author{
Debraj Chatterjee \\ Computer Science and Engineering of TINT \\ (MAKAUT) \\ Techno International Newtown (MAKAUT) \\ Kolkata, India \\ debraj.chatterjee@tict.edu.in
}

\begin{abstract}
From ancient times science and technology have eased human life in all ways. So, it's not surprising that with the advancement of human civilization human needs will be increased and technology has to be developed at the same pace. While meeting these needs and making everyday life easier, better; here comes the importance of robotic arm studies. Robot arms can be controlled by an outside user or by doing predetermined instructions. Nowadays, robot arms are broadly used in the industry and as well the medicine sector. Today, technology is developing at the same pace of rapidly increasing human needs. The work done to meet these needs makes life easier every day, and these studies are concentrated on robotic arm studies. In this project, the robotic arm can move in 4 axis directions with the help of 5 servo motors. With the help of the holder, we can hold the material and move it from one place to another. For doing this, we can connect the android application with the Arduino UNO microcontroller via the Bluetooth module. The abstract part describes why robotic arms have become so important, the introduction part describes types of it, the projected method describes the theory of the project and descriptions of components, lastly how the arm is implemented is described in experimental data and results.
\end{abstract}

Keywords-Robotic arm, Control of robot arm, Motor, Robots, Bluetooth, Arduino Uno, Servo Motor.

\section{INTRODUCTION}

With the advancement of technology, the complexity of work to increases. There are so many times where the work involves risks. Here, come the needs of a machine. A machine can easily and more efficiently do unsafe, repetitive work in much lesser time. The robotic arm can store the sequence of work into its memory and can repeat those when instructed [3]. The term robotics is practically defined as the study, design and use of robot systems for manufacturing. There are two types of robots-service robots and Industry robots. In daily life robots, can do many jobs to make life easier. In industry hazardous work can be done by robots to avoid any accidents. Robots can be easily controlled by the user and are efficient.

There are many types of robotic arms, such as-

According to their Structure [6]: Cartesian robot: It can be used for move the object from one place to another, handling machine tools, arc welding application in various spheres like in assembly operations 2 . Cylindrical robot: It is used for assembly purpose works, handling of machine tools, spot welding. It is a robot that has axes form of a cylindrical coordinate system shows. 3. Spherical robot: It is used for spot welding, fettling machines, gas and arc welding. 4. Articulated robot shows. 5. Parallel robot 6. SCARA robot 7. Anthropomorphic robot: It behaves like a human hand, i.e., independent movement of fingers.
According to their uses [9]: Military robots, Industrial robots, Agricultural robots, Domestic robots, Nanorobots, Swarm Robots, Medical robots, Autonomous drones, Sports Field line Making.

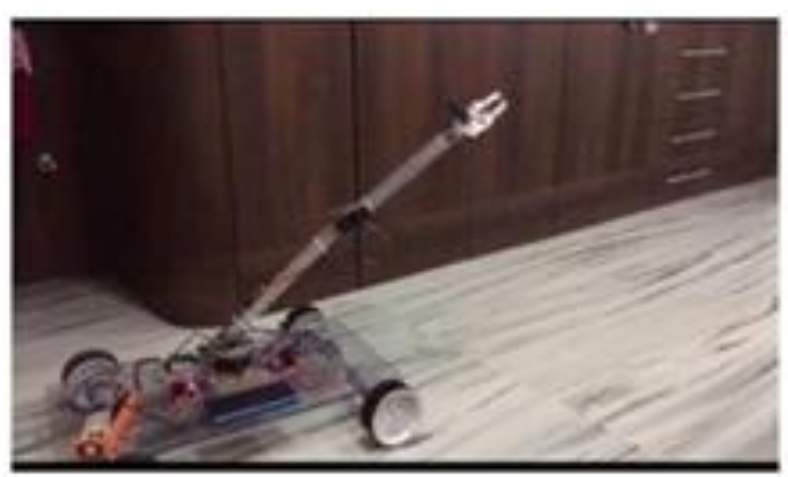

Fig. 1. Bluetooth Controlled Robotic Arm

\section{PROPOSED METHOD}

The requirements of the project are discussed below as main headings and as well as subheadings [5].

\section{A. Components:}

\section{1) Servo Motors.}

What Is A Servo Motor: A servo motor (a linear actuator or rotary actuator) is used to measure precisely the angular position, velocity, and acceleration of an object? Mainly it has many different abilities that generally normal motor does not have. But it can be used as a regular motor and paired with a sensor for position feedback. How Servos Work: Servos use a closed loop mechanism to obtain positional feedback to control linear or rotational speed and position. Both analog and digital signals are accepted by servos. We can use either of it. This electric signal determines the direction and magnitude of movement of the arm as well as the final position of the shaft.

\section{Reasons for using Servo Motor:}

- Operates steadily even at very small or very large speeds and highly sensitive.

- Used in conjunction with electronic or programmable circuits.

- Servos have three cables (red for power, black for ground, yellow for control).

- Controlled by PWM signal at $10-20 \mathrm{~ms}$ and at 0.5 $1.5 \mathrm{~ms}$.

2) Servo Controller 
There are many types of controllers. Among them, servo motor controller is used to control the servo motor. Before selecting a servo motor controller for the industry, it is important to check its compatibility as there are wide varieties of servo motor, controller and servo system.

What Is A Servo Motor Controller?

- The heart of a Servo motor consists of a motor, feedback device and controller.

- The control circuit controls the motion of the motor and the motor drive supplies the power to the motor according to the instructions of the motor controller.

- As servo systems are closed-loop system, it has better transient response time than open-loop system. It also can reduce steady-state errors and the sensitivity of the system.

- Control the motion of a servo and help it to hold a specific position.

- Automatic devices use a feedback mechanism to ensure the output is achieving the desired effect.

- We have used 16 channel Servo Controller (12C bus).

\section{3) Arduino UNO Microcontroller}

In general, PIC is popular in the software and programming sector, but Arduino too is used vigorously nowadays.1) Advanced library can solve complex equations and has an open-source. 2)Fast to execute the programs and plenty of hardware supports are available for work.3) Easy to communicate with the environment.

ATmega328P is used an Arduino Uno. It has 14 digital input/output pins (of which 6 can be used as PWM outputs), 6 analog inputs, a $16 \mathrm{MHz}$ quartz crystal, a USB connection, a power jack, an ICSP header and a reset button. It can be simply connected to a computer with a USB cable or power it with an AC-to-DC adapter or battery to get started. "UNO" is an Italian word that means "ONE". It first released Arduino Software (IDE)1.0. Later this UNO board and version were used as references and evolved. USB Arduino boards' first series is this UNO board.

4) General pin functions [8]:

- LED: Digital pin 13 is connected to the in-built led. The LED will be on if the pin is of high value and the LED will be off if the pin is of low value.

- VIN: When the Arduino board is using an external connection, the input voltage is passed through it.

- $5 \mathrm{~V}$ : It gives an output of $5 \mathrm{~V}$ with the help of the regulator. The power can be transferred through a USB connector, DC power jack or the VIN pin. [where USB $=5 \mathrm{~V}, \mathrm{VIN}=7-20 \mathrm{~V}, \mathrm{DC}=7-20 \mathrm{~V}$ ]. If the voltage is supplied through the regulator via $5 \mathrm{~V}$ or $3.3 \mathrm{~V}$ pins, the board can be damaged.

- 3V3: The on-board regulator generates a 3.3-volt supply. It can draw current up to $50 \mathrm{~mA}$.

- GND: GND is ground pins.

- IOREF: To provide the voltage reference for operating the microcontroller, this pin is used. We need a configured shield to read the IOREF voltage.
Either we have to choose the proper power source or we have to use voltage translators.

- Reset: To shield the block in the board, it is used to add a reset point.

\section{Special pin functions}

- $\quad$ Serial / UART: RX (PIN 0)-receive and TX (PIN 1)accept. These pins are serially connected to the pins of the ATmega8U2 USB-to-TTL chip.

- External interrupts: A trigger can be configured using 2,3 pins to interrupt a rising/falling phase, value change or low value.

- PWM (pulse-width modulation): analog Write() Function provides PWM output with pins $11,10,6,9,5,3$.

- SPI (Serial Peripheral Interface): SPI library supports communication using pins13(SCK), 11(MOSI), 12(MISO) and 10(SS).

- TWI (two-wire interface): Wire library supports TWI and pins SDA (A4), pin SCL (A5) is used.

- AREF : It is analog inputs' reference voltage.

5) Stepper Motor [10]:

- A stepper motor can rotate in equal steps within a full rotation.

- It is a DC electric motor.

- It is also called a Stepping or Step motor.

- It has open-loop controller

- The torque and speed can be controlled without any sensor because the motor's position can be properly controlled.

- The motor can precisely define increment of the shaft as it is dc motor and we know a DC motors rotate continuously until there is supply.

- The "toothed" electromagnets can be activated by microcontroller or driver circuit and it can be seen around gear-shaped iron piece.

- When one magnet is powered on it attracts the iron piece. When the next is turned on, the first becomes already off. Thus, it performs rotations.

- The rotations or steps can be counted in full integer numbers. So, the measurement of the angle is quite precise.

- The arrangement of magnets is divided into groups and the magnets are combinedly called phase. If there are four magnets and two groups (say $\mathrm{C}$ and for example, the circular arrangement will be as following CDCD.

6) Stepper Motor Driver [11]:

- A stepper motor driver controls the stepper motor.

- Before selecting the stepper motor driver, one must ensure the current ratings, voltage ratings to make sure it is compatible with the motor. 
- COMPONENTS: A microcontroller or microprocessor Driver IC Necessary supply of Power.

7) H-Bridge Motor Controller [12]:

Why do we need motor driver IC:1) It switches the polarity of voltage? 2)Allows speed and direction control of two DC motors at the same time.3) Model: L298N.

\section{8) HC -06 Bluetooth Module:}

- HC 06 module transfers data between microcontrollers (bidirectional data transfer) by short-range wireless communication.

- It is connected serially and acts as a slave module and depends on the master module to start the connection. As per the connection the master and slave modules are chosen.

- It has the following pins: Key, VCC, GND, TXD, RXD, STATE.

- It has encryption as well as authentication features as a safety module.

- It is operated in $+3.3 \mathrm{~V}$ to $+6 \mathrm{~V}$ and $40 \mathrm{~mA}$ current.

- It has a Gauss Frequency Shift Keying.

B. Robotic Arm Mechanics

General Characteristics:

- Motion investigation in robotics can be explained by kinematics.

- Denavit and Hardenberg established the general approach to understand the connection of robotic arm links and robot endpoints.

- As per the reference coordinate frame, arm links can be controlled.

- Limb variables and joint coordinates control the linear and angular displacement.

- Forward and reverse kinematics can be seen in robotic arm mechanics

\section{Design Brief:}

The design part is one of the most important parts of the project. It consists of the following sections, such asmechanical part design and mechanical part assemble. It really takes precise hands to join the parts of the robotic arm using tiny microstrip [2]. The following steps have to be performed:

- Select the materials for the project.

- Choose microcontroller and software.

- Collect servo motors, servo motor controller, stepper motor, stepper motor controller.

- Determine and select the parts of the ram.

- Assemble the parts.

- Check the system whether it is compatible with the microcontroller or not.

- If there are any faults correct that part, assemble it and test it again.
- Repeat the last step until there is no error.

1) Mechanical Part Mounting

- Servo motors and the parts are assembled together.

- We have to draw the design first in SOLIDWORKS and then we have started the mounting process.

- The upper part of the arm is movable but the lower part is stationary.

- The lower part can move in the right or left direction with the help of one servo motor (first axis).

- For start and end position two servo motors (second and third axes) are used in the upper part. They operate parallelly to make sure the proper movement of the arm. With the help of these motors, the arm can move in up and down direction.

- The other two servos (fourth and fifth axes) are used for wrist roll, pitch and grip.

2) Robot Arm Control:

1. A connection box has to be made to supply the source to servo motors.

2. Inputs from servo motor, Arduino pin and communication circuit elements are used.

3. To perform the movement of the arm we need to select proper software to connect via Bluetooth and the microcontroller should be taken care of.

4. In" POSITIVE" and "NEGATIVE" directions the arm can be moved with the help of an android application. After the module is paired, it can be modified using the following code to obtain the desired functionality. In this example, we expect the other device (such as an app on a smartphone) to send the command ON or OFF to activate a function on the Arduino.[13]

- Software Serial hc06(Rx, Tx): It defines serial port pins

- hc06.begin(): It defines the baud rate (same value as the module)

- hc06.available(): It tests whether data are available in the buffer of the module or not

- hc06.read(): It reads data one byte at a time

- hc06.print(): It sends a string in ASCII form

- hc06.write(): It sends data one byte at a time

We can connect the microcontroller with routers, Bluetooth, WIFI. Here we are using Bluetooth to connect with mobile and controlling the arm with the app. We can also use a webpage to connect the microcontroller but in that case, it will put a lot of pressure on the microcontroller. Since the microcontroller already has to control the motor, servo and stepper so it will not be a good idea to put more pressure on it. When we are using the app, it makes the mobile as a server and microcontroller as the client. So, in this way, we can control multiple robots like this using the app.

3) How to Operate the Robotic Arm [7]: 


\section{COMPONENTS:}

- Arduino Uno

- Computer (Arduino IDE has to be installed)

- Android smartphone which has the Bluetooth capability

- Bluetooth Module (It is not so costly. Available online)

- THE MIT APP INVENTOR APP (To send instructions to the Arduino via android smartphone).

CONNECTION:

First Step: Arduino Sketch. A simple sketch is needed to do this:

- Arduino and the Bluetooth module should be connected serially.

- Process the input after listening from the port(serial). Second Step: Connecting Bluetooth module with Arduino Uno

The BLUETOOTH module is connected to the Arduino serially. The following are the four pins:

- VCC: Supplies power, connected to $5 \mathrm{v}$ pin of Arduino.

- GND: A ground pin that is connected to the Ground pin of Arduino.

- TXD: Transfers data between module and Arduino and is connected serially with RX (serial receive pin, pin-0 for UNO) of Arduino.

- RXD: Receives data from Arduino and is connected serially with TX (serial transfer pin, pin-1 for UNO) of

- Arduino. "STATE" and the "KEY" pins of the Bluetooth module are not used here.

Third Step: Making the Android phone prepared for the connection:

- MIT APP INVENTOR has to be installed on our phone.

- The Bluetooth must be activated and enabled. Open the settings menu on the phone, select the "Wireless and Networks" category under Bluetooth settings.

\section{Fourth Step: Checking the Connection}

1. Power on the Arduino Uno and the Bluetooth module.

2. Pair it with your phone. The defaults for Bluetooth Module will be:

Default Name: scarlet

Default Pairing code: 1234

3. After pairing the phone and Bluetooth module, select Blueterm. Connect Bluetooth module (default name scarlet) from Blueterm menu. If the connection is successfully established a message will pop out.

\section{EXPERIMENTAL DATA AND RESULTS}

Experimental studies are which we have found through our observations.
Projected Method: At first research has done on robot arms to obtain basic information needed to carry on the project. The robotic arm can move in 4 axes with the help of 5 servo motors. It can move in the right, left, up and down direction. The microcontroller UNO provides control to the system. Stepper motor control is used to control the stepper motors. Stepper motors are used to gain the flexibility of the arm. A Servo motor controller is used to control the servos.

1. Battery: Three lines from battery are going to buck converters. The buck converters are converting the voltages into the following voltages respectively-1) 5 Volt: It goes directly to the microcontroller from the buck converter. 2) 6.6Volt: It splits into two lines-First one goes to the Servo motors. It can rotate up to 16 servos but here we are using only five servos. The second one goes to the Stepper controller.

2. 12Volt: It goes to the Gear motor for driving the bot around.

3. Stepper Motor Controller: We are using only one Stepper controller. Stepper controller can control only one stepper at a time. It has two connections connected to the microcontroller.

4. Servo Motor Controller: We are using four motors and two motor controllers. We are using an L298n motor controller. It has six communication lines, three for each motor. One line is used for speed and the rest two for direction.

5. Android Programming: We have used AppInventor in this project as the android application Inventor: Initially Google and later MIT (Massachusetts Institute of Technology) have developed this free web application. MIT has other this type of applications such as Scratch, StarLogo TNG. To visualize the components of the objects in mobile applications this graphical interface is used. It has visual components such as text fields, pictures, buttons as well as media components (audio, video). It also has acceleration, Bluetooth sensor, GPS. In the design section, we can select the colors, size, position as per our own choice. We create the code which will run in the background in the inventor block section (just like LEGO). Lastly, the blocks must be combined together to get the desired result.

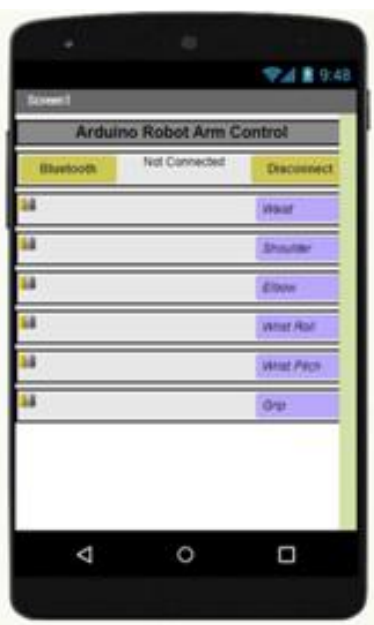

Fig. 2. Android Application 

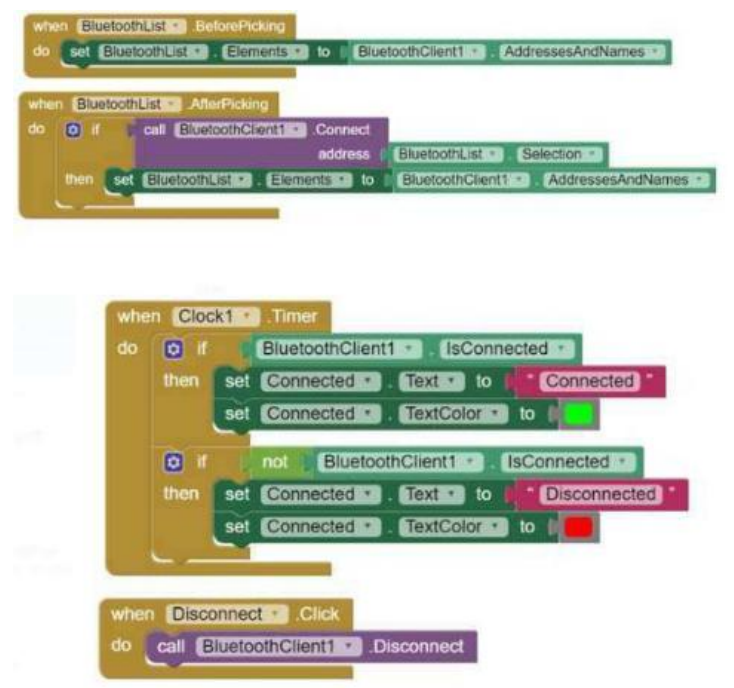

Fig. 3. MIT Inventor App Code I
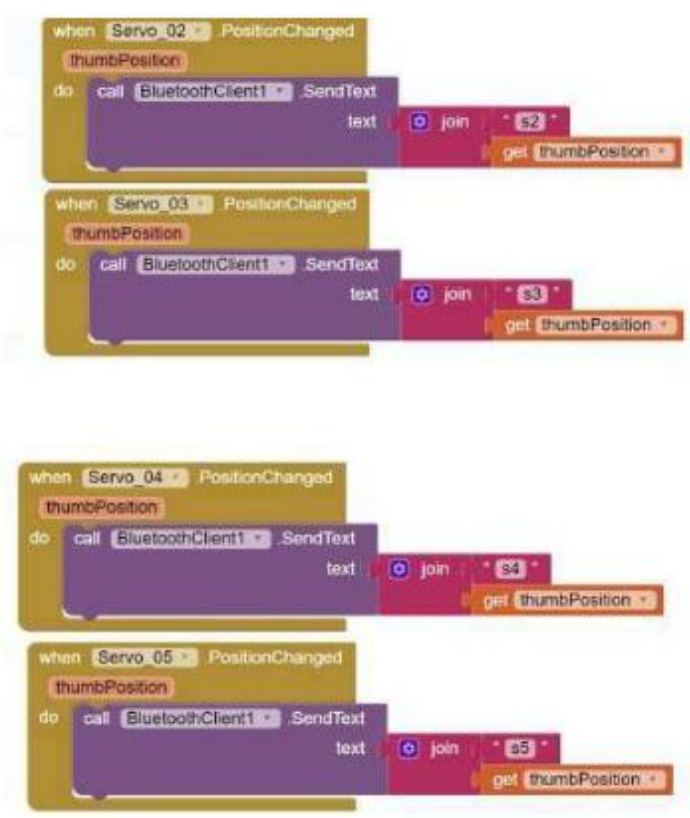

Fig. 4. MIT Inventor App Code II
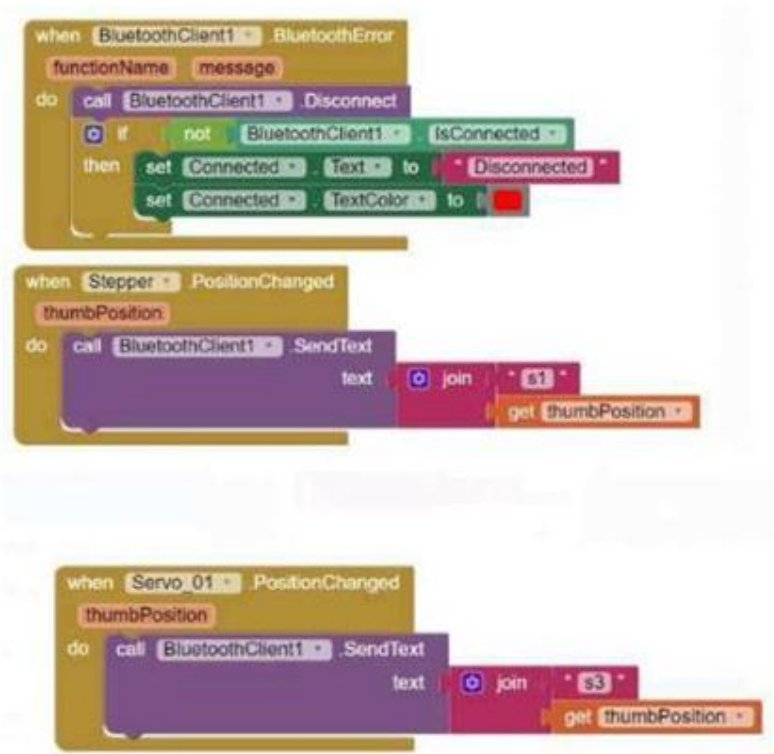

Fig. 5. MIT Inventor App Code III

\section{ADVANTAGES AND DISADVANTAGES ADVANTAGES:}

- To hold and grasp objects it can be used. To mix chemicals which is harmful to humans, it can be used.

- It can be widely used in the laboratory.

- We can dig trenches with it.

- It can be used as a source of entertainment as well as for education purposes.

- It can be used vastly in the medical field to perform critical operations.

- We can make cars with it.

- We can simply handle harmful objects with their help.

\section{DISADVANTAGES:}

- This arm can only pick up small and light objects. Hence it is small-scale production.

- The circuit complexity, as well as the cost will increase if it has to be implemented on a large scale

- If it is implemented largely there is a possibility that the arm can go out of control and make accidents.

\section{FUTURE SCOPE:}

Medical Science - Brain-Computer Interface (BCI) is an immerging field of research, can be used to acquire signals from the human brain and control the arm. They may perform complex operations. Mechanical Design - The arm can be made more efficient, reliable, improved power. Shoulder, elbow \& wrist movement allowing circular \& angular rotations. Universal Gripper - Capable of doing multiple tasks and pick and place any kind of object from one place to another. Intelligence - Capable of making decisions about the task it performs and stores the sequence for future purposes as well as learn from its environment.

\section{CONCLUSION}

Now a day's robotic arms are popular. Though many areas of it still need to be developed thanks to it. It has made work easier and the resulting error level has reduced. The purpose of the project is to provide control of 4 axes moving robot arm design and this robot arm with a suitable microcontroller and Bluetooth module with an android application. The necessary theoretical and practical information for this purpose has been obtained and the necessary infrastructure has been established for the project.

\section{ACKNOWLEDGMENT}

We would like to express our sincere gratitude to Prof. Debraj Chatterjee of the Department of Computer Science Engineering, whose role as project guide was invaluable for the project. We are extremely thankful for the keen interest he took in advising, for the books and reference materials provided for the moral support. Last but not least we convey our gratitude to all the teachers for providing the technical skill that will always remain an asset.

SRIJA DASGUPTA

AVIRUP GHOSH 
Department of Computer Science \& Engineering, Techno International New Town

Kolkata - 700 156, West Bengal, India.

\section{REFERENCES}

[1] WMHW Kadir, RE Samin, BSK Ibrahim. Internet controlled a robotic arm. Procedia Engineering. 2012.

[2] Araújo, D. Portugal, M. Couceiro, C. Figueiredo and R. Rocha, "TraxBot: Assembling and Programming of a Mobile Robotic Platform". In Proc. of the 4th International Conference on Agents and Artificial Intelligence (ICAART 2012), Vilamoura, Portugal, Feb 6-8, 2012.

[3] M. Quigley, B. Gerkey, K. Conley, J. Faust, T. Foote, J. Leibs, E. Berger, R. Wheeler, and A. Y. Ng, "ROS: an open-source Robot Operating System," in Proc. Open-Source Software workshop of the International Conference on Robotics and Automation, Kobe, Japan, May, 2009.

[4] Sciverse Science Direct; Internet Controlled Robotic Arm; Wan Muhamad Hanif Wan Kadira, Reza Ezuan Saminb , Babul Salam Kader Ibrahim (available at www.sciencedirect.com)
[5] Robot Arm Control Using Arduino-by Aimn Mohamed Ahmed Ghiet; RESEARCHGATE-June 2017; DOI: 10.13140/RG.2.2.10227.53286 Affiliation: University of Turkish Aeronautical Association

[6] Internal Journal on Recent Researches In Science, Engineering and Technology-DEVELOPMENT OF ROBOTIC ARM USING ARDUINO UNO- by Priyambada Mishra, Riki Patel, Trushit Upadhyaya, Arpan Desai. Tech Student, Department of Electronics \& Communication, Department of Electronics \& Communication, Charotar University of Science \& Technology, Changa, Gujarat, India. Volume-5, Issue-5, May 2017.

[7] www.google.com

[8] https://en.wikipedia.org/wiki/Arduino_Uno

[9] www.wikipedia.com/robotic

[10] https://en.wikipedia.org/wiki/Stepper_motor

[11] https://www.electrical4u.com/stepper-motor-drive

[12] https://en.wikipedia.org/wiki/H-bridge

[13] https://www.aranacorp.com/en/arduino-and-bluetooth-module-hc-06 